\title{
JEAM: A Novel Model for Cross-Domain Sentiment Classification Based on Emotion Analysis
}

\author{
Kun-Hu Luo, Zhi-Hong Deng*, Liang-Chen Wei, Hongliang Yu \\ School of Electronic Engineering and Computer Science \\ Peking University, Beijing, China \\ \{dr.tiger126@gmail.com, zhdeng@cis.pku.edu.cn, pkuhaywire@ gmail.com, \\ yuhongliang324@gmail.com\}
}

\begin{abstract}
Cross-domain sentiment classification (CSC) aims at learning a sentiment classifier for unlabeled data in the target domain based on the labeled data from a different source domain. Due to the differences of data distribution of two domains in terms of the raw features, the CSC problem is difficult and challenging. Previous researches mainly focused on concepts mining by clustering words across data domains, which ignored the importance of authors' emotion contained in data, or the different representations of the emotion between domains. In this paper, we propose a novel framework to solve the CSC problem, by modelling the emotion across domains. We first develop a probabilistic model named JEAM to model author's emotion state when writing. Then, an EM algorithm is introduced to solve the likelihood maximum problem and to obtain the latent emotion distribution of the author. Finally, a supervised learning method is utilized to assign the sentiment polarity to a given online review. Experiments show that our approach is effective and outperforms state-of-the-art approaches.
\end{abstract}

\section{Introduction}

Cross-domain sentiment classification (CSC) is the task that learns a sentiment classifier for unlabeled data in the target domain based on the labeled data from the source domain. With the increasing amount of opinion information available on the Internet, CSC has become a hot spot in recent years. Traditional machine learning algorithms often train a classifier utilizing the labeled data for CSC. However, in some practical cases, we may have many labeled data for some domains (source domains) but very few or no labeled data for other domains (target domains). Due to the differences of the distribution of two domains in terms of raw features, e.g. raw term frequency, the classifier trained from the source domain often performs badly on the target domain. To overcome this issue, several feature-based studies have been proposed to improve the sentiment classification domain adaptation [Zhuang et al., 2013; He et al., 2011; Gao and Li, 2011; Li et al., 2012; Dai et al., 2007; Zhuang et al., 2010; Pan et al., 2010; Wang et al., 2011; Long et al., 2012; Lin and He, 2009].

Existing studies build various generative models to solve the domain adaptation problems for CSC. In most cases, the models are trained by using the whole corpora without specifying on the sentiment of the texts. For example, [Zhuang et al., 2013] propose a general framework HIDC to mine high-level concepts (e.g. word clusters) across various domains. However, their learned concepts contain many topics not restricted to the sentiment. On the other hand, some researchers focus on the usage of the sentiment in CSC study [Mitra et al., 2013a; Mitra et al., 2013b; He et al., 2011]. [He et al., 2011] modify JST model [Lin and He, 2009] by incorporating word polarity priors through adjusting the topic-word Dirichlet priors. However, they fail to consider the expression differences among various domains.

To overcome the above issues, we employ "emotion", for its ubiquity among domains. The sentiment words in different domains might vary

${ }^{*}$ Corresponding author 
significantly, but the emotion can be effectively transferred. For example, when expressing the emotion "happiness", one uses "bravo" in the domain of sport, while "yummy" in the domain of food. Therefore, we propose an EA framework to model the latent emotions which are commonly contained in subjective articles and expressed by "emotional words". We infer the sentiment polarity of a document based on the emotion state. The hierarchy of EA is composed by four layers:

\section{(1) Sentiment Layer}

Normally, the sentiment of a document is the general opinion towards a certain event or object. For example, a movie review in IMDB might voice the feeling about the movie by a reviewer [Yu et al., 2013].

(2) Emotion Layer

Based on the emotion classification theories in psychology [Plutchik, 2002], the emotion can be classified into the basic ones influenced by the physiological factors, e.g. happiness, sadness, anger, etc., and dozens of complicated ones formed under some specific social conditions, e.g. shame, guilt, abashment, etc. Additionally, the emotion can be classified as positive and negative (similar to the sentiment classification) based on dimensional models of emotion [Schlosberg, 1954; Plutchik, 2002; Rubin and Talerico, 2009]. Intuitively, we assume that a document tends to contain the emotions of similar polarity.

(3) Lexicon Layer

To build the connection between words and the emotion, we introduce emotional words instead of raw word features into our model. By utilizing the emotional lexicon MPQA [Wiebe et al., 2005], we select groups of strong polar words, which get high scores in the emotional lexicon. These words are considered highly correlated to the certain emotion of the same polarity. And these strong polar words have invariant polarity across domains. Therefore, the emotion can be substantialized by a series of emotional words drawn from corresponding probability distribution.

(4) Expression Layer

In many practical cases, data come from different domains. We suppose that the correlation between emotion state and sentiment orientation is stable over domains, but one emotion may have different expressions when domain varies. E.g., "satisfaction" may be expressed as "interesting" or "attractive" for a book; meanwhile, it may be expressed "efficient" for an electronics device. Formally, we have

$$
\begin{gathered}
p(e \mid y, r 1)=p(e \mid y, r 2)=p(e \mid y) \\
p\left(w_{e} \mid e, r 1\right) \neq p\left(w_{e} \mid e, r 2\right)
\end{gathered}
$$

where $e$ denotes the emotion, $y$ denotes the author's sentiment orientation, $r 1$ and $r 2$ denotes two different domains, and $w_{e}$ denotes the emotional words.

Along this line, we propose the Joint Emotion Analysis Model (named JEAM for abbreviation) utilizing the probabilistic methods. See details in the next section.

\section{Proposed Model}

\subsection{Problem Formulation}

The CSC problem can be formulated as follows: Suppose we have two sets of data, denoted as $D_{S}$ and $D_{t}$, which represent the source domain data and the target domain data respectively. In the CSC problem, the source domain data consist of labeled instances, denoted by $D_{s}=$ $\left.\left\{\left(x_{i}^{(s)}, y_{i}^{(s)}\right)\right\}\right|_{i=1} ^{n_{s}}$, where $x_{i}^{(s)} \in \mathbb{R}^{k}$ is an input vector, $y_{i}^{(s)} \in\{0,1\}$ is the output label, and $n_{s}$ is the number of documents in $D_{s}$. Unlike that of the source domain, the target domain data consists of samples without any label information, denoted by $D_{t}=\left.\left\{x_{i}^{(t)}\right\}\right|_{i=1} ^{n_{t}}$, where $x_{i}^{(t)} \in \mathbb{R}^{k}$ is an input vector, and $n_{t}$ is the number of documents in $D_{t}$. The task of CSC is to leverage the training data of source domain $D_{s}$ to predict the label $y_{i}^{(t)}$ corresponding to input vector $x_{i}^{(t)}$ of target domain $D_{t}$.

\subsection{The JEAM Model}

To model the author's emotion state contained in the document, we propose the JEAM model based on the probabilistic graphical principle. Note that all the factors and edges in JEAM are derived from the specific concepts and relations in EA, e.g., $\mathrm{Eq}(1)$ and $\mathrm{Eq}(2)$. We draw the graphical representation of JEAM in Figure 1, and show the notations of this paper in Table 1 .

In Figure 1, $y$ denotes the sentiment orientation of the author, which is a latent variable in this model. $e$ denotes any emotion (topic) generated by $y$ from a conditional probability $p(e \mid y) . e$ is also a latent variable in this model. $r$ denotes any data domain, e.g., books, dvd, kitchen, and electronics etc. $d$ denotes any document chosen from domain $r$ with label y. For documents from the source domain, the conditional probability $p(d \mid r, y)$ is known, which can be used to supervise the modeling process. $u$ denotes the prior sentiment polarity of the corresponding emotional word. In practice, $u$ can be obtained from the emotional lexicon, 


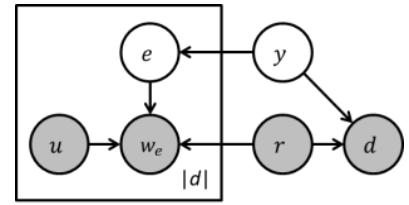

Figure 1. The Graphical representation of JEAM. All the latent variables are marked in white, and all the observed variables are marked in gray.

\begin{tabular}{|c|l|}
\hline$e$ & Emotion \\
$w_{e}$ & Emotional word \\
$r$ & Domain \\
$d$ & Document \\
$u$ & Prior sentiment polarity of the emotional word \\
$y$ & Sentiment polarity of the document \\
$X$ & All the observed variables \\
$\theta$ & All the model parameters \\
\hline
\end{tabular}

Table 1. Means of Symbols

which classifies a series of words into positive and negative categories. $w_{e}$ denotes any emotional word with polarity $u$, which is chosen over words conditioned on emotion $e$ and domain $\mathrm{r}$ from conditional probability $p\left(w_{e} \mid e, r, u\right)$. In this paper, we only select emotional words with strong sentiment polarities to represent the vector of the document. Therefore, we rebuild the data with the help of emotional lexicon cutting out the nonemotional words. As a result, any word chosen from the rebuilt data will be an emotional word, which is supposed not to change its polarity in different domains. Additionally, the joint probability over all the observed variables can be defined as follows based on the hidden variables:

$$
p\left(w_{e}, d, r, u\right)=\sum_{e, y} p\left(e, y, w_{e}, d, r, u\right)
$$

Based on the graphical model, we have: $p\left(e, y, w_{e}, d, r, u\right)=$

$$
p\left(w_{e} \mid e, r, u\right) p(d \mid r, y) p(e \mid y) p(r) p(y) p(u)
$$

We need to learn the unobservable probabilities (e.g., $\quad p\left(w_{e} \mid e, r, u\right), p(d \mid r, y)$, $p(e \mid y), p(y))$ to infer the hidden emotion distribution. Therefore, we develop an Expectation-Maximization (EM) algorithm to maximize the log likelihood of generating the whole dataset and obtain the iterative formula in E-step as follows:

$$
\begin{aligned}
& p\left(e, y \mid w_{e}, d, r, u\right)= \\
& \frac{p\left(w_{e} \mid e, r, u\right) p(d \mid r, y) p(e \mid y) p(r) p(y) p(u)}{\sum_{e, y} p\left(w_{e} \mid e, r, u\right) p(d \mid r, y) p(e \mid y) p(r) p(y) p(u)}
\end{aligned}
$$

where all the factors are calculated in M-step similar to PLSA and HIDC (Hoffman, 1999; Zhuang et al., 2013).

\subsection{CSC via JEAM}

To use JEAM to solve CSC problems, we adopt two optimizations:

First, we supervise the EM optimization with the polarity information of emotional words and instances respectively in the source domain. On the one hand, we estimate $p\left(e, y \mid w_{e}, d, r, u\right)$ utilizing the polarity label of the emotional words. Let the emotion set $E$ be divided into positive set $E_{p}$ and negative set $E_{n}$. We set $p\left(e_{i}, y \mid w_{e}, d, r, u_{w_{e}}\right)=0$ during the whole EM process when the polarities of the emotion and current emotional word are different. On the other hand, we estimate the probability $p(d \mid r, y)$ with the label information of instances in the source domain. When the document is from the source domain, we set $p(d \mid r, y)=0$ if $y$ is different with the ground truth.

Second, we reconstruct the document as follows,

$$
d^{*}=\left[e_{1}, e_{2}, \ldots e_{m}\right], e_{i}=\left\{\begin{array}{c}
1+\frac{\left|W_{e}^{r}\right|}{\sum_{j=1}^{m}\left|W_{e j}^{r}\right|}, i f W_{e_{i}}^{r} \neq \emptyset \\
0, \text { others }
\end{array}\right.
$$

where $\left[e_{1}, e_{2}, \ldots e_{m}\right]$ is the distribution over emotions, $\quad W_{e_{i}}^{r}=\left\{w_{e} \mid e_{w_{e}}^{r}=i\right\} \quad, \quad e_{w_{e}}^{r}=$ $\operatorname{argmax}_{e} p\left(e \mid w_{e}, r\right)$, and $p\left(e \mid w_{e}, r\right)$ can be computed based on $p\left(w_{e} \mid e, r, u\right), p(e \mid y), p(u)$ and $p(r)$ obtained after EM algorithm. The main function of this step is to process a new given document faster, avoiding training JEAM again with the new input. Finally, a machine learning method Support Vector Machine (SVM) is introduced to train a classifier with the labeled data from the source domain and assign polarities to documents from the target domain based on our reconstructed data.

\section{Experiments}

\subsection{Experimental Setup}

We demonstrate the effectiveness of JEAM on the Multi-Domain sentiment data set [Blitzer et al., 2007] which contains four types (domains) of real-world product documents taken from Amazon.com, which are books, dvd, electronics and kitchen. We randomly select 1800 documents from the one domain (source domain) and 200 documents from another domain (target domain). Then, we train a sentiment classifier using documents selected from the source domain and 
assign labels to documents selected from the target domain, which generates 12 classification tasks. We preform 10 random selections and report the average results over 10 different runs. We use MPQA subjective lexicon ${ }^{1}$ as the emotional lexicon. In our experiments, only strongly subjective clues are considered as emotional words, consisting of 1717 positive and 3621 negative words. We rebuild the dataset by cutting out the non-emotional words. For experiment parameters, we set $p=25, n=25$, and $T=100$ after plenty of experiments. Considering the data in practice, the sentiment orientation y has only two forms, positive or negative. Note that we do neither instance selection nor complicated feature selection (only filter the low-frequency words) to our proposed method and other methods in comparison.

\subsection{Experimental Result}

\section{Performance of Emotional Words}

We show the effectiveness of introducing emotional words to solve the CSC problem. In JEAM, we reconstruct the documents by cutting out the non-emotional words. To compare the classification accuracy on the original documents and the reconstructed (emotional) documents, we choose two common classification algorithms, linear SVM and PLSA (topic size=10) for experiment respectively. The experiment results shows that both SVM and PLSA perform better on the emotional documents $(60.43 \%$ and $60.48 \%)$ than on the original documents $(57.73 \%$ and $56.69 \%$ ) for the average accuracy over 12 classification tasks.

\section{Effectiveness of using domain information and word polarity}

We show the effectiveness of using domain information and word polarity, which are employed in our approach. For this purpose, we repeat the experiment without introducing domain and word polarity (node $\mathrm{u}$ and node $\mathrm{r}$ ) into the model. Figure 2 shows the results. As it is clear, the highest performance can be achieved when domain information and word polarity are both used, while the lowest performance is obtained when neither of them is used.

\section{Comparison with the Baselines}

We compare our proposed approach with PLSA, SVM, SFA [Pan et al., 2010], JST [He et al., 2011] and HIDC [Zhuang et al., 2013]. The experimental results of the 12 classification tasks are shown in Figure 3. It can be observed that our

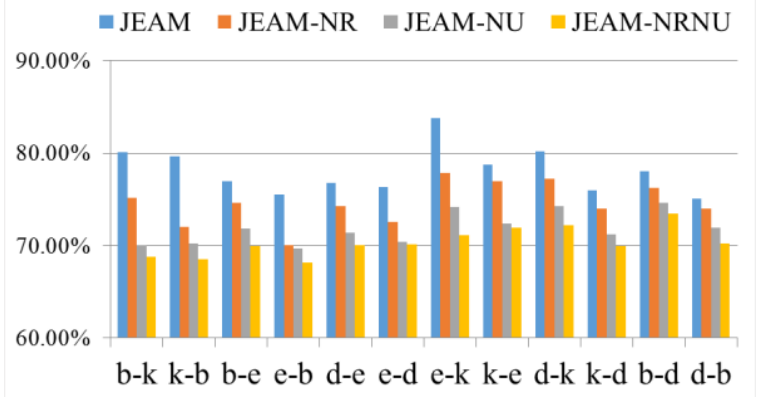

Figure 2: Effectiveness of using domain information and word polarity

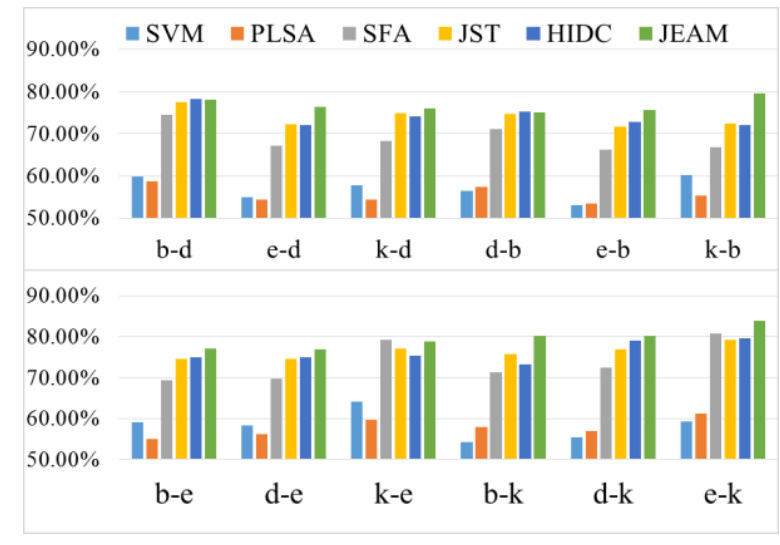

Figure 3: Comparison with the Baselines

proposed approach outperforms all the other approaches in general. Note that in order to obtain a more precise comparison of the algorithms, we do neither the instance selection nor the complicated feature selection. The result of our proposed approach can possibly be improved with the help of these selection strategies.

\section{Conclusion}

In this paper, we propose a novel framework to solve the CSC problem, by modelling emotions across domains. We deeply analyze the relation between the author and the document based on the emotion theories in the field of psychology. Along this line, we propose a framework named EA, which takes the emotions and domains into account. Based on EA, we propose a novel model named JEAM to model the author's emotion state for Cross-domain sentiment classification. We conduct extensive experiments on real datasets to evaluate JEAM. The experiment results show that emotion plays an important role in CSC and JEAM outperforms existing state-of-the-art methods on the task of CSC.

\footnotetext{
${ }^{1}$ http://www.cs.pitt.edu/mpqa
} 


\section{Acknowledgement}

This work is partially supported by Project 61170091 supported by National Natural Science Foundation of China and Project 2015AA015403 supported by the National High Technology Research and Development Program of China (863 Program). We would also like to thank the anonymous reviewers for their helpful comments.

\section{References}

John Blitzer, Mark Dredze, Fernando Pereira, et al.2007. Biographies, bollywood, boom-boxes and blenders: Domain adaptation for sentiment classification. In $A C L$, volume 7, pages 440-447.

Wenyuan Dai, Gui-Rong Xue, Qiang Yang, and Yong Yu. 2007. Co-clustering based classification for outof-domain documents. In Proceedings of the 13th ACM SIGKDD international conference on Knowledge discovery and data mining, pages 210 219. ACM.

Hal Daum'e III. 2009. Frustratingly easy domain adaptation. arXiv preprint arXiv:0907.1815.

Sheng Gao and Haizhou Li. 2011. A cross-domain adaptation method for sentiment classification using probabilistic latent analysis. In Proceedings of the 20th ACM international conference on Information and knowledge management, pages 1047-1052. ACM.

Boqing Gong, Kristen Grauman, and Fei Sha. 2013. Connecting the dots with landmarks: Discriminatively learning domain-invariant features for unsupervised domain adaptation. In Proceedings of The 30th International Conference on Machine Learning, pages 222-230.

Yulan He, Chenghua Lin, and Harith Alani. 2011. Automatically extracting polarity-bearing topics for cross-domain sentiment classification. In Proceedings of the 49th Annual Meeting of the Association for Computational Linguistics: Human Language Technologies-Volume 1, pages 123-131. Association for Computational Linguistics.

Thomas Hofmann. 1999. Probabilistic latent semantic indexing. In Proceedings of the 22nd annual international ACM SIGIR conference on Research and development in information retrieval, pages 5057. ACM.

Shoushan Li, Chu-Ren Huang, Guodong Zhou, and Sophia Yat Mei Lee. 2010. Employing personal/ impersonal views in supervised and semisupervised sentiment classification. In Proceedings of the 48th annual meeting of the association for computational linguistics, pages 414-423. Association for Computational Linguistics.
Fangtao Li, Sinno Jialin Pan, Ou Jin, Qiang Yang, and Xiaoyan Zhu. 2012. Cross-domain co-extraction of sentiment and topic lexicons. In Proceedings of the 50th Annual Meeting of the Association for Computational Linguistics: Long Papers-Volume 1, pages 410-419. Association for Computational Linguistics.

Chenghua Lin and Yulan He. 2009. Joint sentiment/ topic model for sentiment analysis. In Proceedings of the 18th ACMconference on Information and knowledge management, pages 375-384. ACM.

Mingsheng Long, Jianmin Wang, Guiguang Ding, Wei Cheng, Xiang Zhang, and Wei Wang. 2012. Dual transfer learning. In SDM, pages 540-551. SIAM.

Mitra Mohtarami. Man lan, and chew lim tan. 2013a. from semantic to emotional space in probabilistic sense sentiment analysis. In the 27th AAAI Conference on Artificial Intelligence.

Sinno Jialin Pan, Xiaochuan Ni, Jian-Tao Sun, Qiang Yang, and Zheng Chen. 2010. Cross-domain sentiment classification via spectral feature alignment. In Proceedings of the 19th international conference on World wide web, pages 751-760. ACM.

Bo Pang and Lillian Lee. 2008. Opinion mining and sentiment analysis. Foundations and trends in information retrieval, 2(1-2):1-135.

Bo Pang, Lillian Lee, and Shivakumar Vaithyanathan. 2002. Thumbs up?: sentiment classification using machine learning techniques. In Proceedings of the ACL-02 conference on Empirical methods in natural language processing-Volume 10, pages 7986. Association for Computational Linguistics.

Robert Plutchik. 1980. Emotion: A psychoevolutionary synthesis. Harpercollins College Division.

Robert Plutchik. 2002. Emotion and life.

David C Rubin and Jennifer M Talarico. 2009. A comparison of dimensional models of emotion: Evidence from emotions, prototypical events, autobiographical memories, and words. Memory, 17(8):802-808.

Harold Schlosberg. 1954. Three dimensions of emotion. Psychological review, 61(2):81.

Hua Wang, Heng Huang, Feiping Nie, and Chris Ding. 2011. Cross-language web page classification via dual knowledge transfer using nonnegative matrix trifactorization. In Proceedings of the 34th international ACM SIGIR conference on Research and development in Information Retrieval, pages 933-942. ACM.

Janyce Wiebe, Theresa Wilson, and Claire Cardie. 2005. Annotating expressions of opinions and emotions in language. Language resources and evaluation, 39(2-3):165-210. 
Hongliang Yu, Zhi-Hong Deng, and Shiyingxue Li. 2013. Identifying sentiment words using an optimization-based model without seed words. In ACL (2), pages 855-859.

Fuzhen Zhuang, Ping Luo, Peifeng Yin, Qing He, and Zhongzhi Shi. 2013. Concept learning for crossdomain text classification: A general probabilistic framework. In Proceedings of the Twenty-Third international joint conference on Artificial Intelligence, pages 1960-1966. AAAI Press.

Fuzhen Zhuang, Ping Luo, Changying Du, Qing He, Zhongzhi Shi, and Hui Xiong. 2014. Triplex transfer learning: exploiting both shared and distinct concepts for text classification. Cybernetics, IEEE Transactions on, 44(7):1191-1203. 